Ibn Al-Haitham Jour. for Pure \& Appl. Sci. 32 (2) 2019

Ibn Al Haitham Journal for Pure and Applied Science

Journal homepage: http://jih.uobaghdad.edu.iq/index.php/j/index

\title{
Application of Iterative Method for Solving Higher Order Integro- Differential Equations
}

\author{
Samaher M. Yassein \\ Department of Mathematics, College of Education for Pure Science Ibn Al-Haitham, \\ University of Baghdad, Baghdad, Iraq. \\ samamarez@yahoo.com
}

Article history: Received 13 November 2018, Accepted 20 January 2019, Publish May 2019

Doi: $10.30526 / 32.2 .2139$

\begin{abstract}
The main aim of this paper is to apply a new technique suggested by Temimi and Ansari namely (TAM) for solving higher order Integro-Differential Equations. These equations are commonly hard to handle analytically so it is request numerical methods to get an efficient approximate solution. Series solutions of the problem under consideration are presented by means of the Iterative Method (IM). The numerical results show that the method is effective, accurate and easy to implement rapidly convergent series to the exact solution with minimum amount of computation. The MATLAB is used as a software for the calculations.
\end{abstract}

Keywords: Integro-Differential Equations, Iterative Method, Temimi and Ansari method.

\section{Introduction}

Integro-Differential Equations (IDEs) include in many mathematical formulations of physical phenomena, these problems have a major role of interest and arise in many applications in various fields of science, such as chemical kinetics, fluid dynamics, engineering problems and biological models. In recent years, there exist numerical techniques gained a great interest by many authors such as Lagrange Interpolation Method [1]. WaveletGalerkin Method (WGM) [2]. Adomian's Decomposition Method (ADM) [3-5]. Modified Adomian Decomposition Method (MADM) [6]. Variational Iteration Method (VIM) [7, 8]. Differential Transform Method [9]. Tau Method [10]. Generalized Spline Method [11], and Semi Analytical-Numerical Techniques such that Taylor polynomials [12]. and Rationalized Haar Functions Method [13]. However, none of a fore mentioned methods are successfully solved higher order IDEs. Furthermore, prior studies need more effort to realize the outcomes, they are not precise and commonly they are improved for specific sorts IDEs. Temimi and Ansari (TAM) have been suggested a new iterative method, i.e., Semi Analytic Iterative Method (SAIM) for solving linear and nonlinear functional equations [14]. This method has been extensively studied by many researchers recently; it has been successfully applied for 
solving some linear and nonlinear partial and ordinary differential equations [15-18]. It is worth mentioning, SAIM is not yet used to solve higher order IDEs. This method is accurate and powerful technique, needn't to impose any additional restrictions to get the numerical solution of these problems. It is qualified method for extremely the number of calculations will be reduced while still maintaining the solution is more accurate and efficient. Higher Order Linear Fredholm IDEs was solved by Power Series and Chebyshev Series Approximation Methods in [19]. IDEs was solved by Modified Taylor Expansion Method in [20]. And Voltera IDEs was solved by using the Laplace Transform Method in [21]. In this research, we applied this reliable technique to solve higher order IDEs. Generally, Voltera integro - differential equation [21]. Given in the form:

$\mathrm{y}^{(\mathrm{n})}(\mathrm{x})=\mathrm{h}(\mathrm{x})+\lambda \int_{0}^{\mathrm{x}} \mathrm{k}(\mathrm{x}, \mathrm{t}) y(\mathrm{t}) \mathrm{d}(\mathrm{t}), \lambda \neq 0$

And Fredholm - integro - differential equation [19]. given in the form:

$\mathrm{y}^{(\mathrm{n})}(\mathrm{x})$

$=\mathrm{h}(\mathrm{x})+\int_{\mathrm{a}}^{\mathrm{b}} \mathrm{k}(\mathrm{x}, \mathrm{t}) y(\mathrm{t}) \mathrm{d}(\mathrm{t})$

Both of Equations. (1) \& (2) with Initial Conditions (ICs).

$\mathrm{y}^{(\mathrm{k})}(0)=\omega_{\mathrm{k}}, \quad 0 \leq \mathrm{k} \leq \mathrm{n}-1$

where $\mathrm{y}^{(\mathrm{n})}(\mathrm{x})$ is the $\mathrm{n}^{\text {th }}$ derivatives, $\mathrm{k}(\mathrm{x}, \mathrm{t})$ and $\mathrm{h}(\mathrm{x})$, are given continuous smooth functions, $\lambda$ is a parameter, $y(\mathrm{x})$ unknown function to be determined and $a, b, \omega_{\mathrm{k}}$ are constants. Since the results of equation (1) and equation (2) combine the differential and integral operators, then it is necessary to define ICs as in (3). The proposed method was applied to establish series solutions for Equation (1) or Equation (2). We illustrated that this method is effective and perfect in handling to solve higher order IDEs in scientific and engineering problems. Several numerical examples are introduced and comparison with existing methods, the results reveal that the method is accurate and easy to implement.

\section{Fundamental Idea for the Iterative Method}

The main steps of Iterative Method (IM). It is rewrite that any differential equation can be written as [14].

$\mathrm{L}(\mathrm{y}(\mathrm{x}))+\mathrm{N}(\mathrm{y}(\mathrm{x}))+\mathrm{h}(\mathrm{x})=0$

With Boundary Conditions $\mathrm{B}\left(\mathrm{y}, \frac{d y}{d x}\right)=0$,

Where $\mathrm{x}$ is the independent variable, $\mathrm{L}$ is a Linear operator, $\mathrm{N}$ is a non-linear operator and the boundary operator is B. The method which proposed as the following way. The initial approximation is the primary step in the IM, by assuming that the initial guess $y_{0}(\mathrm{x})$ is solution of problem $\mathrm{y}(\mathrm{x})$ and solution of equation can be solving:

$\mathrm{L}\left(y_{0}(\mathrm{x})\right)+\mathrm{h}(\mathrm{x})=0, \mathrm{~B}\left(y_{0}, \frac{d y_{0}}{d x}\right)=0$

To generate the next iteration of the solution as follows:

$\mathrm{L}\left(y_{1}(\mathrm{x})\right)+\mathrm{h}(\mathrm{x})+\mathrm{N}\left(y_{0}(\mathrm{x})\right)=0, \mathrm{~B}\left(y_{1}, \frac{d y_{1}}{d x}\right)=0$ 
After several simple iterative steps of the solution, the general form of this Equation which is:

$\mathrm{L}\left(\mathrm{y}_{\mathrm{n}+1}(\mathrm{x})\right)+\mathrm{h}(\mathrm{x})+\mathrm{N}\left(\mathrm{y}_{\mathrm{n}}(\mathrm{x})\right)=0, \mathrm{~B}\left(\mathrm{y}_{\mathrm{n}+1}, \frac{\mathrm{dy} \mathrm{n}+1}{\mathrm{dx}}\right)=0$

Evidently each iteration of the function $y_{n}(\mathrm{x})$ represent effectively alone solution for equation (4).

We will implement the steps of method at the equation (1), so Equation (1) can be express as:

$\mathrm{L}(\mathrm{y}(\mathrm{x}))=\mathrm{h}(\mathrm{x})+\lambda \int_{0}^{\mathrm{x}} \mathrm{k}(\mathrm{x}, \mathrm{t}) y(\mathrm{t}) \mathrm{d}(\mathrm{t}), \quad \lambda \neq 0$,

The differential operator $\mathrm{L}(\mathrm{y}(\mathrm{x}))$ is the highest order derivative in the equation (8), we assume that $\mathrm{L}$ is invertible by using the given ICs in equation (3) and applying the inverse operator $\mathrm{L}^{-1}$ in both sides of equation (8), we get the following Equation:

$\mathrm{y}(\mathrm{x})=\psi_{0}+\mathrm{L}^{-1}(\mathrm{~h}(\mathrm{x}))+\mathrm{L}^{-1}\left(\lambda \int_{0}^{\mathrm{x}} \mathrm{k}(\mathrm{x}, \mathrm{t}) \mathrm{y}(\mathrm{t}) \mathrm{d}(\mathrm{t})\right), \quad \lambda \neq 0$,

Where the function $\psi_{0}$ is arising from integrating the source term, from applying the given ICs in Equation (3) which are prescribed.

Now, we illustrate the method as the following steps:

Step 1: to get $y_{0}(t)$ solving $\mathrm{L}\left(y_{0}(\mathrm{x})\right)-\mathrm{h}(\mathrm{x})=0$

with ICs in Equation (3) and applying the inverse operator $\mathrm{L}^{-1}$ in both sides of Equation (10), we obtain:

$\mathrm{y}_{0}(x)=\psi_{0}+\mathrm{L}^{-1}(\mathrm{~h}(\mathrm{x}))$

Step 2: The next iterate is:

$\mathrm{L}\left(\mathrm{y}_{1}(\mathrm{x})\right)-\mathrm{h}(\mathrm{x})-\int_{0}^{\mathrm{x}} \mathrm{k}(\mathrm{x}, \mathrm{t}) \mathrm{y}_{0}(\mathrm{t}) \mathrm{d}(\mathrm{t})=0 \quad$ with ICs in Eq. (3) ,

solving this equation and applying the inverse operator $\mathrm{L}^{-1}$ in both sides of Equation (11), leads to get $y_{1}(x)$ as:

$\mathrm{y}_{1}(x)=\psi_{0}+\mathrm{L}^{-1}(\mathrm{~h}(\mathrm{x}))+\mathrm{L}^{-1}\left(\int_{0}^{\mathrm{x}} \mathrm{k}(\mathrm{x}, \mathrm{t}) \mathrm{y}_{0}(\mathrm{t}) \mathrm{d}(\mathrm{t})\right)$

Step (3): After several simple iterative steps of the solution, the general form of this equation given as

$$
L\left(y_{n+1}(t)\right)-h(x)-\int_{0}^{x} k(x, t) y_{n}(t) d(t)=0 \quad \text { with ICs in Eq. (3) }
$$

Solving this equation and applying the inverse operator $\mathrm{L}^{-1}$ in both sides of Equation (12), leads to get $y_{n+1}(x)$ :

$\mathrm{y}_{\mathrm{n}+1}(x)=\psi_{0}+\mathrm{L}^{-1}(\mathrm{~h}(\mathrm{x}))+\mathrm{L}^{-1}\left(\int_{0}^{\mathrm{x}} \mathrm{k}(\mathrm{x}, \mathrm{t}) \mathrm{y}_{\mathrm{n}}(\mathrm{t}) \mathrm{d}(\mathrm{t})\right)$

evidently each iteration of the function $y_{n}$ (x) represents effectively solution for Equation (8). Similarly, by the same steps we solve Fredholm IDEs. 
Ibn Al-Haitham Jour. for Pure \& Appl. Sci. 32 (2) 2019

\section{Numerical Results}

We will be applying the SAIM for solving some examples of the Fredholm IDEs and Voltera IDEs.

\section{Example 1}

Consider third- order Fredholm IDE [19]:

$\mathrm{y}^{(3)}(\mathrm{x})=1-\mathrm{e}+\mathrm{e}^{\mathrm{x}}+\int_{0}^{1} \mathrm{y}(\mathrm{t}) \mathrm{dt}$,

with ICs $\mathrm{y}(0)=\mathrm{y}^{\prime}(0)=\mathrm{y}^{\prime \prime}(0)=1$, the Exact solution is $\mathrm{Y}(\mathrm{x})=\mathrm{e}^{\mathrm{x}}$

\section{Solution}

Via implementing same steps as described in the previous section, we first begin by solving the following initial problem to find the initial approximation $y_{0}(\mathrm{x})$, the SAIM will be applied as

$\mathrm{L}\left(\mathrm{y}_{0}\right)=1-\mathrm{e}+\mathrm{e}^{\mathrm{x}}$ with $y_{0}(0)=y_{0}{ }^{\prime}(0)=y_{0}^{\prime \prime}(0)=1$ where $\mathrm{h}(\mathrm{x})=\mathrm{e}-\mathrm{e}^{\mathrm{x}}-1, \mathrm{~L}(\mathrm{y})=\frac{\mathrm{d}^{3} \mathrm{y}}{\mathrm{dx}^{3}}$, $\mathrm{N}(\mathrm{y})=0$.

So, the primary step is:

$\mathrm{L}\left(\mathrm{y}_{0}\right)=1-\mathrm{e}+\mathrm{e}^{\mathrm{x}}$ with $\quad y_{0}(0)=y_{0}{ }^{\prime}(0)=y_{0}^{\prime \prime}(0)=1$

Then, the general relation as follows:

$$
L\left(y_{n+1}\right)-h(x)-\int_{0}^{x} k(x, t) y_{n}(t) d(t)=0, y_{n+1}(0)=y^{\prime}{ }_{n+1}(0)=y^{\prime \prime}{ }_{n+1}(0)=1
$$

By solving the problem defined in Equation (14), we have $\quad \mathrm{y}_{0}=\mathrm{e}^{\mathrm{x}}-\frac{1934613350591413}{6755399441055744} \mathrm{x}^{3}$ The first iteration can be gotten as:

$\mathrm{y}_{1}{ }^{(3)}(\mathrm{x})=1-\mathrm{e}+\mathrm{e}^{\mathrm{x}}+\int_{0}^{1} \mathrm{y}_{0}(\mathrm{t}) \mathrm{dt}$ with $y_{1}(0)=\mathrm{y}^{\prime}{ }_{1}(0)=\mathrm{y}^{\prime \prime}{ }_{1}(0)=1$

Thus, the solution of Equation (16) as: $\quad \mathrm{y}_{1}=\mathrm{e}^{\mathrm{x}}-\frac{644871116863805}{54043195528445952} \mathrm{x}^{3}$.

The second iteration is:

$\mathrm{y}_{2}{ }^{(3)}(\mathrm{x})=1-\mathrm{e}+\mathrm{e}^{\mathrm{x}}+\int_{0}^{1} \mathrm{y}_{1}(\mathrm{t}) \mathrm{dt}$ with $y_{2}(0)=y_{2}{ }^{\prime}(0)=y^{\prime \prime}{ }_{2}(0)=1$

Then, the solution of Eqaution (17) as $\mathrm{y}_{2}=\mathrm{e}^{\mathrm{x}}-\frac{26869629869327}{54043195528445952} \mathrm{x}^{3}$.

Also, by same steps, the other solutions can be generated from calculating these problems via using MATLAB, we obtain $\mathrm{y}_{3}, \mathrm{y}_{4}, \ldots$, we get the solution till $\mathrm{y}_{11}$.

$\mathrm{y}_{11}=\mathrm{e}^{\mathrm{x}}-\frac{11}{54043195528445952} \mathrm{x}^{3}$ 
Ibn Al-Haitham Jour. for Pure \& Appl. Sci. 32 (2) 2019

Table 1. Numerical results of the illustrative example above of $\mathrm{Y}_{11}$.

\begin{tabular}{|c|c|c|c|}
\hline $\boldsymbol{x}$ & Exact solution & SAIM & Error \\
\hline 0 & 1.000000000000000 & 1.000000000000000 & 0 \\
\hline 0.1 & 1.105170918075648 & 1.105170918075648 & 0 \\
\hline 0.2 & 1.221402758160170 & 1.221402758160170 & 0 \\
\hline 0.3 & 1.349858807576003 & 1.349858807576003 & 0 \\
\hline 0.4 & 1.491824697641270 & 1.491824697641270 & 0 \\
\hline 0.5 & 1.648721270700128 & 1.648721270700128 & 0 \\
\hline 0.6 & 1.822118800390509 & 1.822118800390509 & 0 \\
\hline 0.7 & 2.013752707470477 & 2.013752707470477 & 0 \\
\hline 0.8 & 2.225540928492468 & 2.225540928492468 & 0 \\
\hline 0.9 & 2.459603111156950 & 2.459603111156950 & 0 \\
\hline 1 & 2.718281828459046 & 2.718281828459046 & 0 \\
\hline
\end{tabular}

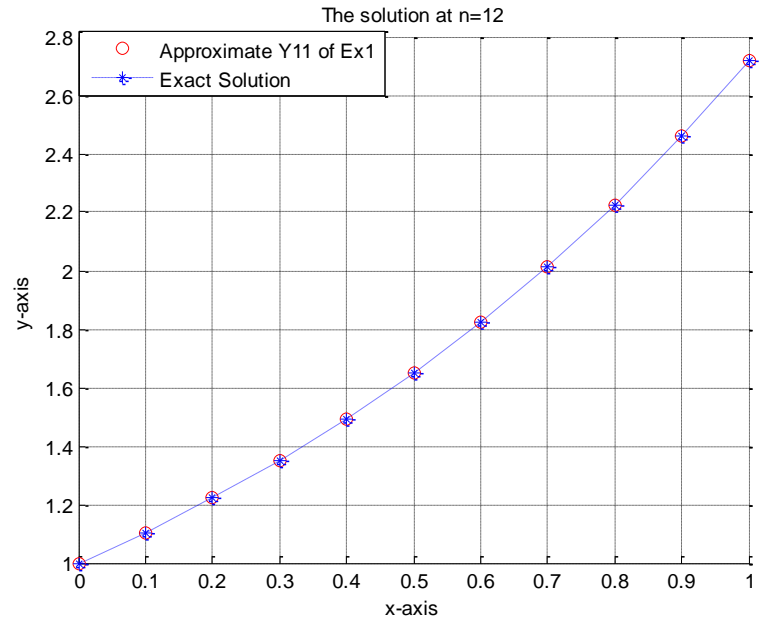

Figure 1. Exact and approximate solution of the illustrative example.

\section{Example 2}

Consider third- order Voltera IDE [20].

$\mathrm{y}^{(3)}(\mathrm{x})-\mathrm{xy}^{\prime \prime}(\mathrm{x})=\frac{4}{7} \mathrm{x}^{9}-\frac{8}{5} \mathrm{x}^{7}-\mathrm{x}^{6}+6 \mathrm{x}^{2}-6+4 \int_{0}^{\mathrm{x}} \mathrm{x}^{2} \mathrm{t}^{3} \mathrm{y}(\mathrm{t}) \mathrm{dt}$

With ICs $\mathrm{y}(0)=1, \mathrm{y}^{\prime}(0)=2, \mathrm{y}^{\prime \prime}(0)=0, \mathrm{x} \geq 0, t \leq 1$,

The Exact solution is $Y(x)=-x^{2}+2 x+1$,

\section{Solution}

Applying same steps as in the previous example, we first begin by solving the following initial problem in order to find the initial approximation $y_{0}(\mathrm{x})$, the SAIM will be applied: as $\mathrm{L}\left(\mathrm{y}_{0}\right)=\frac{4}{7} \mathrm{x}^{9}-\frac{8}{5} \mathrm{x}^{7}-\mathrm{x}^{6}+6 \mathrm{x}^{2}-6$ with $y_{0}(0)=1, y_{0}{ }^{\prime}(0)=2, y_{0}{ }^{\prime \prime}(0)=0$ where $h(x)=-\frac{4}{7} x^{9}+\frac{8}{5} x^{7}+x^{6}-6 x^{2}+6, L(y)=\frac{d^{3} y}{d x^{3}}, N(y)=0$.

So, the primary step is:

$\mathrm{L}\left(\mathrm{y}_{0}\right)=\frac{4}{7} \mathrm{x}^{9}-\frac{8}{5} \mathrm{x}^{7}-\mathrm{x}^{6}+6 \mathrm{x}^{2}-6$ with $y_{0}(0)=1, y_{0}^{\prime}(0)=, y_{0}^{\prime \prime}(0)=0$

Then, the general relation as follows:

$\mathrm{L}\left(\mathrm{y}_{\mathrm{n}+1}\right)-\mathrm{h}(\mathrm{x})-\int_{0}^{\mathrm{x}} \mathrm{k}(\mathrm{x}, \mathrm{t}) \mathrm{y}_{\mathrm{n}}(\mathrm{t}) \mathrm{d}(\mathrm{t})=0, \mathrm{y}_{\mathrm{n}+1}(0)=1, \mathrm{y}_{\mathrm{n}+1}^{\prime}(0)=2, \mathrm{y}^{\prime \prime}{ }_{\mathrm{n}+1}(0)=0$

By solving the problem defined in Equation (19) we have: 
$y_{0}=\frac{x^{12}}{2310}-\frac{x^{10}}{450}-\frac{x^{9}}{504}+\frac{x^{5}}{10}-x^{3}+2 x+1$

by same way in the previous example. The next step is to find $y_{1}$ as follows:

The first iteration can be gotten as:

$\mathrm{y}_{1}{ }^{(3)}(\mathrm{x})=\frac{4}{7} \mathrm{x}^{9}-\frac{8}{5} \mathrm{x}^{7}-\mathrm{x}^{6}+6 \mathrm{x}^{2}-6+\mathrm{xy}_{0}{ }^{\prime \prime}(\mathrm{x})+4 \int_{0}^{\mathrm{x}} \mathrm{x}^{2} \mathrm{t}^{3} \mathrm{y}_{0}(\mathrm{t}) \mathrm{dt}(21)$ and has solution

$\mathrm{y}_{1}=\frac{\mathrm{x}^{21}}{73735200}-\frac{\mathrm{x}^{19}}{9157050}-\frac{\mathrm{x}^{18}}{8019648}+\frac{4 \mathrm{x}^{14}}{85995}-\frac{\mathrm{x}^{12}}{6600}-\frac{\mathrm{x}^{11}}{6930}+\frac{\mathrm{x}^{7}}{105}-\mathrm{x}^{3}+2 \mathrm{x}+1$ and

$\mathrm{y}_{2}=\frac{x^{30}}{11226184200000}-\frac{x^{28}}{1034948105100}-\frac{x^{27}}{774096523200}+\frac{20747 x^{23}}{13750604627760}-\frac{709 \mathrm{x}^{21}}{75209904000}-$

$\frac{1657 x^{20}}{147891744000}+\frac{31 x^{16}}{8731800}-\frac{x^{14}}{109200}-\frac{x^{13}}{108108}+\frac{x^{9}}{1260}-x^{3}+2 x+1$.

Hence, in iteration steps, we have:

$y_{12}=$

$3.062817340869851 \times 10^{-82} \times x^{120}-1.101677695323877 \times 10^{-80} \times x^{118}-2.725562818772886 \times$ $10^{-80} \times x^{117}+1.778958719423882 \times 10^{-75} \times x^{113}-5.682824661052544 \times 10^{-74} \times x^{111}-$

$1.370653337095081 \times 10^{-73} \times x^{110}+3.984172272372894 \times 10^{-69} \times x^{106}-1.119818251782719 \times$ $10^{-67} \times x^{104}-2.628843074816043 \times 10^{-67} \times x^{103}+4.503028638092516 \times 10^{-63} \times x^{99}-$

$1.101417450504745 \times 10^{-61} \times x^{97}-2.511985562347862 \times 10^{-61} \times x^{96}+2.827909775701136 \times$ $10^{-57} \times x^{92}-5.940775524869664 \times 10^{-56} \times x^{90}-1.313503431540083 \times 10^{-55} \times x^{89}+$ $1.026299389853779 \times 10^{-51} \times x^{85}-1.822336772542073 \times 10^{-50} \times x^{83}-3.896422990960605 \times$ $10^{-50} \times x^{82}+2.175609109040211 \times 10^{-46} \times x^{78}-3.200678509627877 \times 10^{-45} \times x^{76}-$ $6.598849111192931 \times 10^{-45} \times x^{75}+2.671403340873855 \times 10^{-41} \times x^{71}-3.173731777895746 \times$ $10^{-40} \times x^{69}-6.287576987469821 \times 10^{-40} \times x^{68}+1.85332923203967 \times 10^{-36} \times x^{64}$ $1.718197579672121 \times 10^{-35} \times x^{62}-3.257269778930915 \times 10^{-35} \times x^{61}+1.027480158702644 \times$ $10^{-49} \times x^{59}+6.961493020520868 \times 10^{-32} \times x^{57}-4.798561225077398 \times 10^{-31} \times x^{55}-$ $8.659823953316347 \times 10^{-31} \times x^{54}+3.114021333121303 \times 10^{-44} \times x^{52}+1.32543948187801 \times$ $10^{-27} \times x^{50}-6.307772348188415 \times 10^{-27} \times x^{48}-1.076556205716984 \times 10^{-26} \times x^{47}+$ $2.671419265527734 \times 10^{-39} \times x^{45}+1.154719813291063 \times 10^{-23} \times x^{43}-3.330521444892846 \times$ $10^{-23} \times x^{41}-5.329596586395636 \times 10^{-23} \times x^{40}+7.831725504493517 \times 10^{-35} \times x^{38}+$ $3.873151195225192 \times 10^{-20} \times x^{36}-4.991934045359734 \times 10^{-20} \times x^{34}-7.402102900997027 \times$ $10^{-20} \times x^{33}+7.642681309794397 \times 10^{-31} \times x^{31}+3.461646293785214 \times 10^{-17} \times x^{29}+$ $2.098836527787579 \times 10^{-27} \times x^{24}+1.058401144288997 \times 10^{-24} \times x^{17}-x^{3}+2 x+1$

Table 2. Numerical results of the illustrative example above of $Y_{12}$.

\begin{tabular}{|c|c|c|c|}
\hline $\boldsymbol{x}$ & Exact solution & SAIM & Error \\
\hline 0 & 1.000000000000000 & 1.000000000000000 & 0 \\
\hline 0.1 & 1.199000000000000 & 1.199000000000000 & 0 \\
\hline 0.2 & 1.392000000000000 & 1.392000000000000 & 0 \\
\hline 0.3 & 1.573000000000000 & 1.573000000000000 & 0 \\
\hline 0.4 & 1.736000000000000 & 1.736000000000000 & 0 \\
\hline 0.5 & 1.875000000000000 & 1.875000000000000 & 0 \\
\hline 0.6 & 1.984000000000000 & 1.984000000000000 & 0 \\
\hline 0.7 & 2.057000000000000 & 2.057000000000000 & 0 \\
\hline 0.8 & 2.088000000000000 & 2.088000000000000 & 0 \\
\hline 0.9 & 2.071000000000000 & 2.071000000000000 & 0 \\
\hline 1 & 2.000000000000000 & 2.000000000000000 & 0 \\
\hline
\end{tabular}


Ibn Al-Haitham Jour. for Pure \& Appl. Sci. 32 (2) 2019

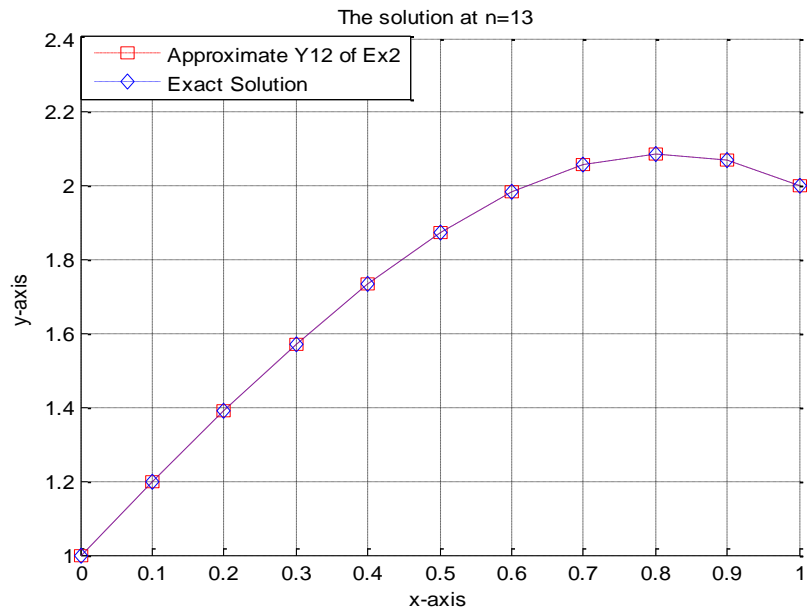

Figure 2. Exact and approximate solution of the illustrative example.

\section{Example 3}

Consider fourth - order Fredholm IDE [19, 22].

$\mathrm{y}^{(4)}(\mathrm{x})=\frac{1}{4}+(1-2 \ln 2) \mathrm{x}-\frac{6}{(1+\mathrm{x})^{4}}+\int_{0}^{1}(\mathrm{x}-\mathrm{t}) \mathrm{y}(\mathrm{t}) \mathrm{dt}$

with ICs $\mathrm{y}(0)=0, \mathrm{y}^{\prime}(0)=1, \mathrm{y}^{\prime \prime}(0)=-1, \mathrm{y}^{\prime \prime \prime}(0)=2$,

The exact solution $\mathrm{Y}(\mathrm{x})=\ln (1+\mathrm{x})$.

\section{Solution}

To find $\mathrm{y}_{0}$ we take $\mathrm{L}\left(\mathrm{y}_{0}\right)=\frac{1}{4}+(1-2 \ln 2) \mathrm{x}-\frac{6}{(1+\mathrm{x})^{4}}$ with $\mathrm{y}_{0}(0)=0, \mathrm{y}_{0}{ }^{\prime}(0)=$ $1, \mathrm{y}_{0}{ }^{\prime \prime}(0)=-1, \mathrm{y}_{0}{ }^{\prime \prime \prime}(0)=2$ where $\mathrm{h}(\mathrm{x})=\frac{1}{4}+(1-2 \ln 2) \mathrm{x}-\frac{6}{(1+\mathrm{x})^{4}}, \mathrm{~L}(\mathrm{y})=\frac{\mathrm{d}^{4} \mathrm{y}}{\mathrm{dx}^{4}}, \mathrm{~N}(\mathrm{y})=$ 0 . So, the primary step is:

$\mathrm{L}\left(\mathrm{y}_{0}\right)=\frac{1}{4}+(1-2 \ln 2) \mathrm{x}-\frac{6}{(1+\mathrm{x})^{4}}$ with $y_{0}(0)=0,{y_{0}}^{\prime}(0)=1, y_{0}{ }^{\prime \prime}(0)=-1, y_{0}{ }^{\prime \prime}{ }^{\prime(0)}=2$

Then, the general relation as:

$\mathrm{L}\left(\mathrm{y}_{\mathrm{n}+1}\right)-\mathrm{h}(\mathrm{x})-\int_{0}^{\mathrm{x}} \mathrm{k}(\mathrm{x}, \mathrm{t}) \mathrm{y}_{\mathrm{n}}(\mathrm{t}) \mathrm{d}(\mathrm{t})=0, \mathrm{y}_{\mathrm{n}+1}(0)=0, \mathrm{y}_{\mathrm{n}+1}^{\prime}(0)=1, \mathrm{y}_{\mathrm{n}+1}^{\prime \prime}(0)=-1, \mathrm{y}^{\prime \prime \prime}{ }_{\mathrm{n}+1}(0)=2$

By solving the problem defined in Equation (22) we have:

$\mathrm{y}_{0}=\ln (\mathrm{x}+1)+\frac{\mathrm{x}^{4}}{96}-\frac{579905046931621}{180143985094819840} \mathrm{x}^{5}$

By same way the next step is to find $y_{1}$ as follows: The first iteration can be gotten as:

$y_{1}{ }^{(4)}(\mathrm{x})=\frac{1}{4}+(1-2 \ln 2) \mathrm{x}-\frac{6}{(1+\mathrm{x})^{4}}+\int_{0}^{1}(\mathrm{x}-\mathrm{t}) y_{0}(\mathrm{t}) \mathrm{dt}$

and has a solution:

$$
\begin{aligned}
& y_{1}=\ln (\mathrm{x}+1)-\frac{3621025736840333}{68094426365841899520} x^{4}+\frac{104493422922097}{8106479329266892800} x^{5} \\
& y_{2}=\ln (\mathrm{x}+1)+\frac{398426818305727}{1361888527316837990400} x^{4}-\frac{2407976480303}{34047213182920949760} x^{5}
\end{aligned}
$$


Ibn Al-Haitham Jour. for Pure \& Appl. Sci. 32 (2) 2019

Hence, in iteration steps, we have:

$y_{7}=\ln (\mathrm{x}+1)-\frac{1446180158675437}{980744084899180960278380544000000} x^{4}+\frac{977445743089}{1539253760479322796195840000000} x^{5}$.

Table 3. Numerical results of the illustrative example above of $Y_{7}$.

\begin{tabular}{|c|c|c|c|}
\hline $\boldsymbol{x}$ & Exact solution & SAIM & Error \\
\hline 0 & 0 & 0 & 0 \\
\hline 0.1 & 0.095310179804325 & 0.095310179804325 & 0 \\
\hline 0.2 & 0.182321556793955 & 0.182321556793955 & 0 \\
\hline 0.3 & 0.262364264467491 & 0.262364264467491 & 0 \\
\hline 0.4 & 0.336472236621213 & 0.336472236621213 & 0 \\
\hline 0.5 & 0.405465108108164 & 0.405465108108164 & 0 \\
\hline 0.6 & 0.470003629245736 & 0.470003629245736 & 0 \\
\hline 0.7 & 0.530628251062170 & 0.530628251062170 & 0 \\
\hline 0.8 & 0.587786664902119 & 0.587786664902119 & 0 \\
\hline 0.9 & 0.641853886172395 & 0.641853886172395 & 0 \\
\hline 1 & 0.693147180559945 & 0.693147180559945 & 0 \\
\hline
\end{tabular}

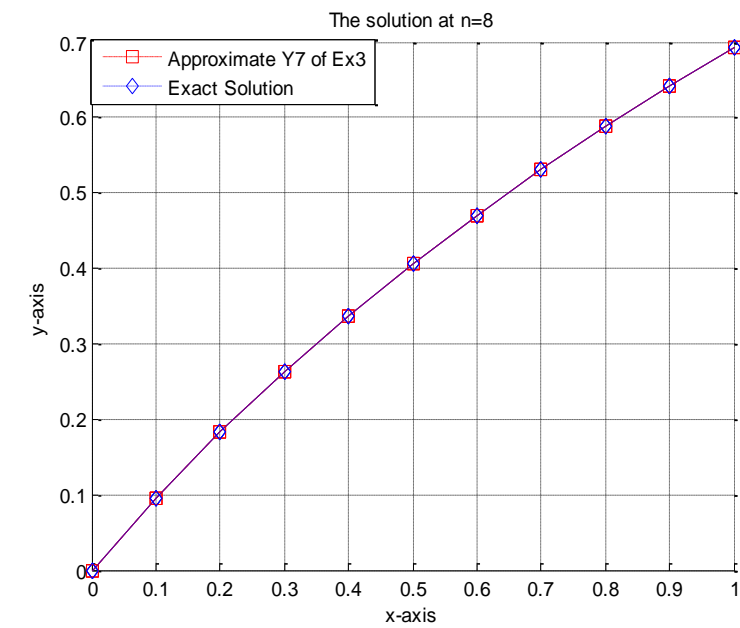

Figure 3. Exact and approximate solution of the illustrative example.

\section{Example 4}

Finally, consider fourth - order Voltera-IDE [21, 23].

$\mathrm{y}^{(4)}(x)=\sin \mathrm{x}+\cos \mathrm{x}+2 \int_{0}^{\mathrm{x}} \sin (\mathrm{x}-\mathrm{t}) \mathrm{y}(\mathrm{t}) \mathrm{dt}$

with ICs $\mathrm{y}(0)=\mathrm{y}^{\prime}(0)=\mathrm{y}^{\prime \prime}(0)=\mathrm{y}^{\prime \prime \prime}(0)=1$, the Exact solution $\quad \mathrm{Y}(\mathrm{x})=\mathrm{e}^{\mathrm{x}}$

\section{Solution}

To find $y_{0}$ we take $\mathrm{L}\left(\mathrm{y}_{0}\right)=\sin \mathrm{x}+\cos \mathrm{x}$ with $\mathrm{y}(0)=\mathrm{y}^{\prime}(0)=\mathrm{y}^{\prime \prime}(0)=\mathrm{y}^{\prime \prime \prime}(0)=1$, where $g(x)=-\sin x-\cos x, L(y)=\frac{d^{4} y}{d x^{4}}, N(y)=0$. So, the primary step is taken:

$$
\mathrm{L}\left(\mathrm{y}_{0}\right)=\sin \mathrm{x}+\cos \mathrm{x}, \quad \mathrm{y}_{0}(0)=\mathrm{y}_{0}{ }^{\prime}(0)=\mathrm{y}_{0}{ }^{\prime \prime}(0)=\mathrm{y}_{0}{ }^{\prime \prime \prime}(0)=1
$$

Then, the general relation as:

$$
L\left(y_{n+1}\right)-h(x)-\int_{0}^{x} k(x, t) y_{n}(t) d(t)=0, y_{n+1}(0)=y_{n+1}^{\prime}(0)=y^{\prime \prime}{ }_{n+1}(0)=y^{\prime \prime \prime}{ }_{n+1}(0)=1
$$


By solving the problem defined in Eq. (26) we have $y_{0}=\cos x+\sin x+x^{2}+\frac{x^{3}}{3}$ as same way, the next step is to find $\mathrm{y}_{1}$ as follows: The first iteration can be gotten as:

$\mathrm{y}_{1}{ }^{(4)}(x)=\sin \mathrm{x}+\cos \mathrm{x}+2 \int_{0}^{\mathrm{x}} \sin (\mathrm{x}-\mathrm{t}) \mathrm{y}(\mathrm{t}) \mathrm{dt}$

and has a solution:

$y_{1}=9 \cos x-8 x+10 \sin x-x \cos x+x \sin x+4 x^{2}+\frac{4 x^{3}}{3}-\frac{x^{4}}{6}-\frac{x^{5}}{30}+\frac{x^{6}}{180}+\frac{x^{7}}{1260}-8$

$\mathrm{y}_{2}=97 \cos \mathrm{x}-96 \mathrm{x}+\frac{223 \sin \mathrm{x}}{2}-\frac{\mathrm{x}^{2} \cos \mathrm{x}}{2}-\frac{\mathrm{x}^{2} \sin \mathrm{x}}{2}-\frac{29 x \cos x}{2}+\frac{27 x \sin x}{2}+36 x^{2}+12 x^{3}-2 x^{4}-\frac{2 x^{5}}{5}+$ $\frac{2 x^{6}}{45}+\frac{2 x^{7}}{315}-\frac{x^{8}}{2520}-\frac{x^{9}}{22680}+\frac{x^{10}}{453600}+\frac{x^{11}}{4989600}-96$.

Table 4. Numerical results of the illustrative example above of $Y_{2}$

\begin{tabular}{|c|c|c|c|}
\hline$x$ & Exact solution & SAIM & Error \\
\hline 0 & 1.000000000000000 & 1.000000000000000 & 0 \\
\hline 0.1 & 1.105170918075648 & 1.105170918075658 & $1.0 \mathrm{E}^{-014}$ \\
\hline 0.2 & 1.221402758160170 & 1.221402758160139 & $3.0 \mathrm{E}^{-014}$ \\
\hline 0.3 & 1.349858807576003 & 1.349858807575984 & $1.9 \mathrm{E}^{-014}$ \\
\hline 0.4 & 1.491824697641270 & 1.491824697641278 & $7 . \mathrm{E}^{-015}$ \\
\hline 0.5 & 1.648721270700128 & 1.648721270700122 & $6 . \mathrm{E}^{-015}$ \\
\hline 0.6 & 1.822118800390509 & 1.822118800390498 & $1.0 \mathrm{E}^{-014}$ \\
\hline 0.7 & 2.013752707470477 & 2.013752707470502 & $2.5 \mathrm{E}^{-014}$ \\
\hline 0.8 & 2.225540928492468 & 2.225540928492492 & $2.4 \mathrm{E}^{-014}$ \\
\hline 0.9 & 2.459603111156950 & 2.459603111156937 & $1.2 \mathrm{E}^{-014}$ \\
\hline 1 & 2.718281828459046 & 2.718281828459041 & $4 . \mathrm{E}^{-015}$ \\
\hline
\end{tabular}

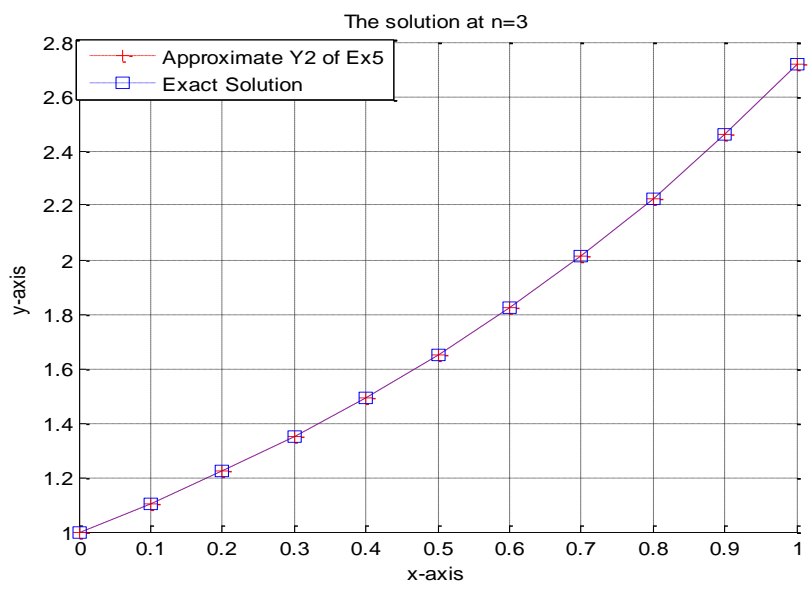

Figure 4. Exact and approximate solution of the illustrative example.

The essence of this method, SAIM in comparison with the other analytical methods does not need large computations such as Lagrange multiplier in the VIM or any complex assumptions like nonlinear Adomian's polynomials in the ADM. It also does not need to constrict Homotopy in the HPM. Furthermore, this method proved that it is efficient in overcoming the difficulties in calculating and solving high -order integro-differential equations with easier steps.

\section{Conclusion}

Most integro-differential equations are difficult to be solved analytically. For this purpose, the efficient Iterative analytic method (Temimi and Ansari) is applied to solve such 
mentioned type of these equations. The present iteration method has strength features among other analytic methods. The obtained solutions can be shown as a series form that converges to the exact solution with simple computations. No complicated calculations have been shown with the presented method. Finally, the advantage of this method is to apply some examples to show that the present iterative method is very accurate and suitable for solving the highorder Voltera - Fredholm Integro-Differential Equations.

\section{References}

1. Rashed, M.T. Lagrange interpolation to compute the numerical solutions of differential, integral and integro-differential equations. Applied Mathematics and Computation.2004,151,869-878,doi: org/10.1016/S0096-3003(03)00543-5.

2. Avudainayagam, A.; Vani, C. Wavelet-Galerkin method for integro-differential Equations. Applied Numerical Mathematics.2000,32,247-254,doi:org/10.1016/ S01689274(99) 00026-4.

3. El-Sayed, S.M.; Abdel-Aziz, M.R. A comparison of Adomian's decomposition method and Wavelet-Galerkin method for integro-differential equations. Applied Mathematics and Computation.2003,136,151-159.

4. Hashim, I. Adomian decomposition method for solving BVPs for fourth-order integro differential equations. Journal of Computational and Applied Mathematics.2006, 193,658-664.

5. Wazwaz, A.M. A reliable algorithm for solving boundary value problems for higherorder integro-differential equations. Applied Mathematics and Computation. 2001,118,327-342, doi: 10.1016/S0096-3003(99)00225-8.

6. Abdul Razzaq, E.A.L.; Yassein, S.M. Analytic Solutions For Integro-Differential Inequalities Using Modified Adomian Decomposition Method. Ibn Al-Haitham Jour for Pure \& Appl. Sci.2017,30,1,177-191.

7. Sweilam, N.H. Fourth order integro-differential equations using variational iteration method. Computers and Mathematics with Applications.2007,54,1086-1091,doi: org/10.1016/j.camwa.2006.12.055.

8. He, J.H. Variational iteration method: New development and applications. Computers and Mathematics with Applications.2007,54,881-894,doi: org /10.1016/ j.camwa. 2006.12.083.

9. Arikoglu, A.; Ozkol, I. Solution of boundary value problems for integro-differential equations by using differential transform method. Applied Mathematics and Computation.2005,168,1145-1158, doi: 10.1016/j.amc.2004.10.009.

10. Hosseini, S.M.; Shahmorad, S. Tau numerical solution of Fredholm integro-differential equations with arbitrary polynomial bases. Applied Mathematical Modeling.2003,27,145154.

11. Hasan, N.N.; Hussien, D.A. Generalized Spline Approach for Solving System of Linear Fractional Volterra Integro-Differential Equations. Ibn Al-Haitham Jour. for Pure \& Appl. Sci.2018,31,1,222-230.

12. Maleknejad, K.; Mahmoudi, Y. Taylor polynomial solution of high-order nonlinear Volterra_Fredholm integro-differential equations. Applied Mathematics and Computation. 2003, 145, 641-653, doi:10.1016/S0096-3003(03)00152-8. 
13. Maleknejad, K.; Mirzaee, F.; Abbasbandy, S. Solving linear integro-differential equations system by using rationalized Haar function method. Applied Mathematics and Computation. 2004, 155, 317-328, doi:10.1016/S0096- 3003(03)00778-1.

14. Temimi, H.; Ansari, A. A Semi Analytical Iterative Technique for Solving Nonlinear Problems. Computers and Mathematics with Applications. 2011, 61, 2, 203-210, doi: org/10.1016/j.camwa.2010.10.042.

15. Farshad, E.; Amin, H.; Frzad, E.; Rohoallah, M. An Iterative Method for Solving Partial Differential Equations and Solution of Korteweg-Devries Equations for Showing the Capability of the Iterative Method. World Applied Programming.2013,3, 4,320-327.

16. Temimi, H.; Ansari, A. A New Iterative Technique for Solving Nonlinear Second Order Multi-Point Boundary Value Problems. Applied Mathematics and Computation. 2011, 218, 4, 1457- 1466, doi:org/10.1016/j.amc.2011.06.029.

17. Nejad, M.Z.; Rastgoo, A.; Hadi, A. Effect of Exponentially-Varying Properties on Displacements and Stresses in Pressurized Functionally Graded Thick Spherical Shells with Using Iterative Technique. Journal of Solid Mechanics. 2014, 6, 4, 366-377.

18. Temimi, H.; Ansari, H.A. Computational Iterative Method for Solving Nonlinear Ordinary Differential Equations. LMS J. Comput. Math. 2015, 18, 1, 730-753, doi: org/10.1112/S1461157015000285.

19. Gegele, O.A.; Evans, O.P.; Akoh, D. Numerical Solution of Higher Order Linear Fredholm - Integro - Differential Equations. American Journal of Engineering Research (AJER). 2014, 3, 8, 243-247.

20. Rashidinia, J.; Tahmasebi, A. Approximate solution of linear integro-differential equations by using modified Taylor expansion method. World Journal of Modelling and Simulation. 2013, 9, 4, 289-301.

21. Wazwaz, A.M. Chapter 5. Linear and Nonlinear Integral Equations Methods and Applications. Saint Xavier University Chicago, USA, Springer. 2011, ISBN 978-3-64221448-6,175- 189.

22. Tawfiq, L.N.M.; Yassien. S.M. Solution of High Order Ordinary Boundary Value Problems Using Semi-Analytic Technique. Ibn Al-Haitham Jour. for Pure \& Appl. Sci. 2013, 26, 1

23. Tawfiq, L.N.M.; Hussein, R.W. On Solution of Regular Singular Initial Value Problems. Ibn Al-Haitham Jour. for Pure \& Appl. Sci. 2013, 26, 1 .

24. Tawfiq, L.N.M.; Rasheed, H.W. On Solution of Regular Singular Ordinary Boundary Value Problem. Ibn Al-Haitham Jour. for Pure \& Appl. Sci. 2013, 26, 2, 249-256. 